\title{
Locomotion asymmetry in young Standardbred trotters in training and links to future
} racing career

\author{
A. Jansson ${ }^{*}$, S. Ringmark, L. Johansson and L. Roepstorff \\ Department of Anatomy, Physiology and Biochemistry, P.O. Box 7011, 75007 Uppsala, Sweden; anna.jansson@slu.se
}

Received: 23 August 2021 / Accepted: 18 October 2021

(๑) 2022 Wageningen Academic Publishers

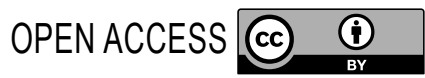

RESEARCH ARTICLE

\begin{abstract}
This study evaluated whether front and hind limb symmetry patterns observed from 1.5 through 3 years of age in Standardbred trotters in training affect racing activity at older age. The hypothesis tested was that asymmetries observed during this period are linked to reduced number of lifetime races and low earnings. Sixteen Standardbreds were subjected to a controlled training and dietary programme from the age of 1.5 years until December as 3-year-olds (P1) and then continued their career with other trainers. On 17 occasions during P1, locomotion asymmetry (vector sum) in front and hind limbs during trot in hand was recorded with a sensor-based system. By 10 years of age, data on lifetime number of races and earnings of the horses were obtained from the Swedish Trotting Association. Horses were divided in groups based on the number of lifetime races performed, i.e. more or less than the median of the cohort born the same year. Mean asymmetry levels did not differ between horses performing more or less lifetime races than the cohort median. However, horses that showed the poorest lifetime race activity showed elevated hind limb asymmetry in August at the age of 3 years, compared to horses with more races (group <median: $9 \pm 6 \mathrm{~mm}$ vs group >median: $6 \pm 2 \mathrm{~mm}(P=0.02)$ ). There were no correlations between individual mean and peak asymmetries and lifetime earnings. It is concluded that locomotion symmetry observed during trot in hand at the age of 1.5-year-olds to December as 3-year-olds was not a simple and straight-forward predictor of future racing career. However, hind limb asymmetries of around $10 \mathrm{~mm}$ in the late season as 3-year-olds could be a warning of impaired future performance.
\end{abstract}

Keywords: earnings, lameness, symmetry, performance

\section{Introduction}

The most common health problem in racing horses is lameness (Dyson et al., 2008; Vigre et al., 2002), which can affect $26-70 \%$ of Standardbred race horses (Gaustad et al., 1996; Vigre et al., 2002). These findings indicate that there is a lack of knowledge about optimal training programmes and about how individual horses should be monitored to minimise the risk of injuries. Early-stage detection can be important for prevention of the development of severe musculoskeletal disorders. Although locomotion asymmetry as measured does not say anything about the cause, it is still an important indication of possible pain from the locomotion apparatus. Instead of subjective methods to assess locomotion asymmetries, the use of objective systems which quantifies the asymmetries has become more common. Objective locomotion analysis systems could thus be used systematically by trainers to monitor locomotion patterns and to assist in tuning the training of individual horses. To enable this, validated methods to uncover important signs of lameness are necessary.

Our research group has performed the only controlled, long-term (2.5 years) study to date on the effect of different training strategies on the performance and health of 1.5-3 year old Standardbred horses (Ringmark et al., 2013, 2015, 2016, 2017a,b). Throughout the 2.5 years, with start in 2010, we regularly used objective locomotion analysis to monitor locomotion symmetry in the horses. Data are now available enabling us to look for links between the symmetry patterns we observed 2010-2012 and the lifetime racing career of the horses. Of special interest during the 2.5 years, are two distinct asymmetry peaks observed in the front limbs (Ringmark et al., 2016). The cause and long-term effect 
remains to be investigated and interestingly, by the end of the 2.5 years, the front limb asymmetry was reduced and no longer different from the levels observed in the horses at breaking at 1.5 years of age. Although there was no overall effect of time on hind limb asymmetry during the 2.5 years, the symmetry pattern differed from that of the front limbs (Ringmark et al., 2016). As soon as short bouts of speed training (typically $200 \mathrm{~m}$ ) on an oval track were introduced a few days per week, at the age of 1.5 years, hind limb asymmetry started to increase and an elevated level was maintained throughout the study (Ringmark et al., 2016). Although the horses were regularly examined by a veterinarian (Ringmark et al. 2016), it was unclear whether this reflected undetected/subclinical lameness or whether it was a normal functional response in Standardbred trotters. Harness racing is not a symmetrical sport, since the horses run at high speed in one direction on an oval track, so asymmetric hind limb locomotion can perhaps be expected. The horses studied have now completed most of their racing career and we have a unique opportunity to investigate if the locomotion asymmetries observed at the age of 1.5 to 4 was a good predictor of horses' lifetime racing career. The aim of the present study was to evaluate whether the front and hind limb asymmetry patterns observed from 1.5 to 4 years affected horses' lifetime racing activity. If the asymmetries observed were mainly present in horses with low number of lifetime races and earnings, locomotion asymmetry at an early age could be an important indicator of pathological conditions that affect the lifetime performance.

\section{Materials and methods}

The first part of the study (2.5 years, age 1.5 until December as 3-year-olds in the horses) was performed at the Swedish National Centre for Trotting Education in Wången, central Sweden and data for the remaining part of the study was collected after horses had been sold to other trainers (7 years, until horses turned 11 years). The research protocol was approved by Umeå Local Ethics Committee (A 90-10, 14 September 2010). Other results from the study have been published previously (Ringmark et al., 2013, 2015, 2016, 2017a,b), and more experimental details can be found in those publications. A description of materials and methods relevant for this paper is provided below.

\section{Horses and management during the first 2.5 years}

Sixteen Standardbred colt yearlings were used. All horses but one entered the study in September 2010 as 1-yearolds (the remaining horse entered in late October 2010). The horses were all castrated in December 2010 or January 2011 and all were fed the same diet throughout the study (Ringmark et al., 2013, 2017a). Horses had ad libitum access to water and haylage and, depending on the nutrient content of the haylage batch, the diet was supplemented with pelleted lucerne (Lantmännen Krafft
AB, Malmö, Sweden), a commercial mineral supplement (Lantmännen $\mathrm{Krafft} \mathrm{AB}$ ) and $\mathrm{NaCl}$, to meet all known nutrient requirements of horses in training (NRC, 2007). The horses were stabled individually for approximately $14 \mathrm{~h}$ per day Monday to Thursday/Friday, and spent the rest of the time in a paddock with access to shelters. Hoof trimming and shoeing was performed every 5-6 weeks and during wintertime (October/November-March) studs ( $8 \mathrm{~mm}$ high) were fitted on the shoes (four per shoe).

\section{Training and health documentation}

All horses were subjected to a controlled, pre-scheduled training programme planned by a group of professional trainers and conducted by one of these trainers. The training programme is described in detail in Ringmark et al. $(2013,2015)$. When the horses were considered sufficiently fit (trainer's opinion), they participated in a preparation race as 2-year-olds, in a qualification race as 3-year-olds and finally in official races.

All training sessions and health concerns (acute or more subtle or subjective) were recorded on individual level. Horses had a veterinary check-up if the driver three times in a row scored the impression of the horse $\geq 2$ (scale $1-3$, 1 = good, 2 = slightly asymmetric but trainable, $3=$ too asymmetric to train) and for acute causes (Ringmark et al., 2016).

\section{Regular recording of locomotion asymmetry}

On 17 occasions, between September as 1-year-olds and December as 3-year-olds, locomotion asymmetry in front and hind limbs was recorded during trot in hand on a packed gravel road for $\sim 100 \mathrm{~m}$. This was done using the sensor-based system Lameness Locator (Equinosis LLC, Columbia, MO, USA), which is similar to the system described by Keegan et al. (2011). The recordings were made immediately after leaving the box, but for some horses a few attempts had to be made before a proper measurement could be performed, due to horse behaviour. The differences in left versus right maximal and minimum position of head and pelvis were used to calculate the vector sum (VS) as: $\sqrt{ }\left(\right.$ maximum difference ${ }^{2}+$ minimum difference ${ }^{2}$ ), for head (front limbs, $\mathrm{VS}_{\text {front }}$ ) and pelvis (hind limbs, $\mathrm{VS}_{\text {hind }}$ ). As mentioned, there was no difference in locomotion symmetry patterns between the two training groups throughout the study (Ringmark et al., 2016).

To increase the understanding of how objective locomotion analysis during trot in hand reflects the locomotion pattern during exercise at higher speed on a track we also performed registrations on the track with horses driven in cart. This was done in May-October as 2-year-olds and all 16 horses were measured two or three times (total of 35 observations). At most there were three days between the measurement 
of locomotion asymmetry in hand and the corresponding measurement performed on the track. Track registrations were performed on a straight, firm, gravel track (similar to a racetrack) for about $200 \mathrm{~m}$ at two speeds; slow and fast. For slow speed the driver aimed at $6 \mathrm{~m} / \mathrm{s}$ and for the fast speed, the driver was instructed to increase speed as much as possible without the horse starting to canter. Speed was registered using a GPS provided by Pegasus Limb Phasing system (European Technology for Business Ltd., Codicote, $\mathrm{UK}$ ) and was $6.0 \pm 0.2 \mathrm{~m} / \mathrm{s}$ (slow) and $8.0 \pm 0.9 \mathrm{~m} / \mathrm{s}$ (fast, means \pm standard deviation). Due to technical problems there was only speed data from 18 of 35 observations.

\section{Follow-up on racing career}

In December 2012 all horses were sold or returned to their breeders, except for one which was euthanised in October 2012 due to lameness. With all remaining horses, attempts were made to continue the career at different trainers. For the purposes of the present study, data on the number of races in which the horses had started by the age of 10 were extracted from the Swedish Trotting Association database in 2019.

The racing career of the experimental horses was evaluated by comparison with the performance of all geldings born in the same year (2009) and registered with a trainer at the age of 2 years $(n=1,183$, data from the Swedish Trotting Association). The number of races performed by the gelding cohort born in 2009 did not show a normal distribution and the median number of lifetime races was 29 . On the 16 experimental horses, locomotion asymmetry in horses that performed more or less than 29 lifetime races (M29 and L29, respectively) was compared. Group M29 included seven horses and group L29 included nine horses.

Data on lifetime earnings and best race record were also obtained from the Swedish Trotting Association. Since Swedish Standardbred horses compete over several distances and with different starting methods, some records were corrected to a race distance of $1,640 \mathrm{~m}$ and autostart. Correction involved deducting $1 \mathrm{~s} / \mathrm{km}$ if the best record was obtained at $2,140 \mathrm{~m}$, or if the race was started without autostart, which is in the range observed in Swedish harness races (Solé et al., 2020).

\section{Statistical analyses}

Statistical analyses were performed using SAS (Version 9.4; Cary, NC, USA). A mixed model including effects of group (lower (L29) or more (M29) than the median), date and interaction between group and date was used; $Y_{i j k}=\mu+\alpha_{i}+\beta_{j}+\alpha_{i} \beta_{j}+a_{k}+e_{i j k}$. $Y_{i j k}$ is the symmetry observation ( $\mathrm{VS}_{\text {front }}$ or $\mathrm{VS}_{\text {hind }}$ ), $\mu$ the mean value, $\alpha_{\mathrm{i}}$ the fixed effect of group, $\beta_{j}$ the fixed effect of date, $\alpha_{i} \beta_{j}$ the interaction between group and date, $a_{n}$ the random effect of horse, and $\mathrm{e}_{\mathrm{ijk}}$ the residuals: $\mathrm{e}_{\mathrm{ijk}} \sim\left(0, \delta^{2}\right)$. Horse was considered a repeated variable. Hand vs track analyses were carried out in R (v3.6.0, R Core Team, 2019) using packages nlme (v3.1-139) and emmeans (v1.3.4). A mixed model was used which included effect of date and group, where horse were considered random and repeated. Data were log-transformed before analysis. Differences were considered significant at $P<0.05$. Values presented are mean \pm standard deviation unless otherwise stated. Pearson's correlation test was used to test whether asymmetry data were correlated to earnings and race record.

\section{Results}

\section{General observations on asymmetry}

There were no differences in $\mathrm{VS}_{\text {hind }}$ or $\mathrm{VS}_{\text {front }}$ between group M29 and L29 ( $P=0.67$ and $P=0.48$, respectively). There was also no interaction between group and date for $\mathrm{VS}_{\text {hind }}$ or $\mathrm{VS}_{\text {front }}(P=0.46$ and $P=0.96$, respectively). However, for both $\mathrm{VS}_{\text {hind }}$ and $\mathrm{VS}_{\text {front, }}$, there was an effect of date $(P=0.02$ and $P<0.0001$, respectively) (Figures 1 and 2 ). There were no differences between groups (Table 1) or significant interactions when data were limited to the recordings collected from July to December at the age of three (the time of intensive race participation).

\section{Changes over time}

There was a significant peak in front limb asymmetry in May as 2-year-olds (M29 group: $20 \pm 16 \mathrm{~mm}$ and L29 group: $24 \pm 18 \mathrm{~mm}$, Figure 1). In groups M29 and L29, the increase was 1.9-fold and 2.4-fold, respectively in May compared with the previous observation in March. In March to June as 3-year-olds, there was another increase in front limb symmetry in both groups, but by the end of the three years season asymmetry levels had decreased in both groups and were similar to those at the start, at age 1.5 years (Figure 1 ).

In M29 horses, hind limb asymmetry was elevated in January at age 2 years $(9 \pm 6 \mathrm{~mm}$, Figure 2$)$. In this group another five significant elevations were observed throughout the study (Figure 2) but by the end of the study no difference compared with the initial levels at age 1.5 years was observed (Figure 2). In group L29, hind limb asymmetry was elevated in May $(9 \pm 5 \mathrm{~mm})$. The asymmetry then decreased, but by the end of the three years season an increase in asymmetry was observed again in L29 (Aug: $9 \pm 6 \mathrm{~mm}$; Aug-Dec: $9 \pm 5 \mathrm{~mm}$, Figure 2).

\section{Relationship to earnings and race record}

There were no significant correlations $(P>0.05)$ between lifetime earnings and mean or peak hind- and front limb asymmetries (Figure 3). There were also no significant correlations $(P>0.05)$ between best racing record (at 2,140 m volt start $(n=12)$ or corrected to 1,640 m autostart $(n=13))$ 


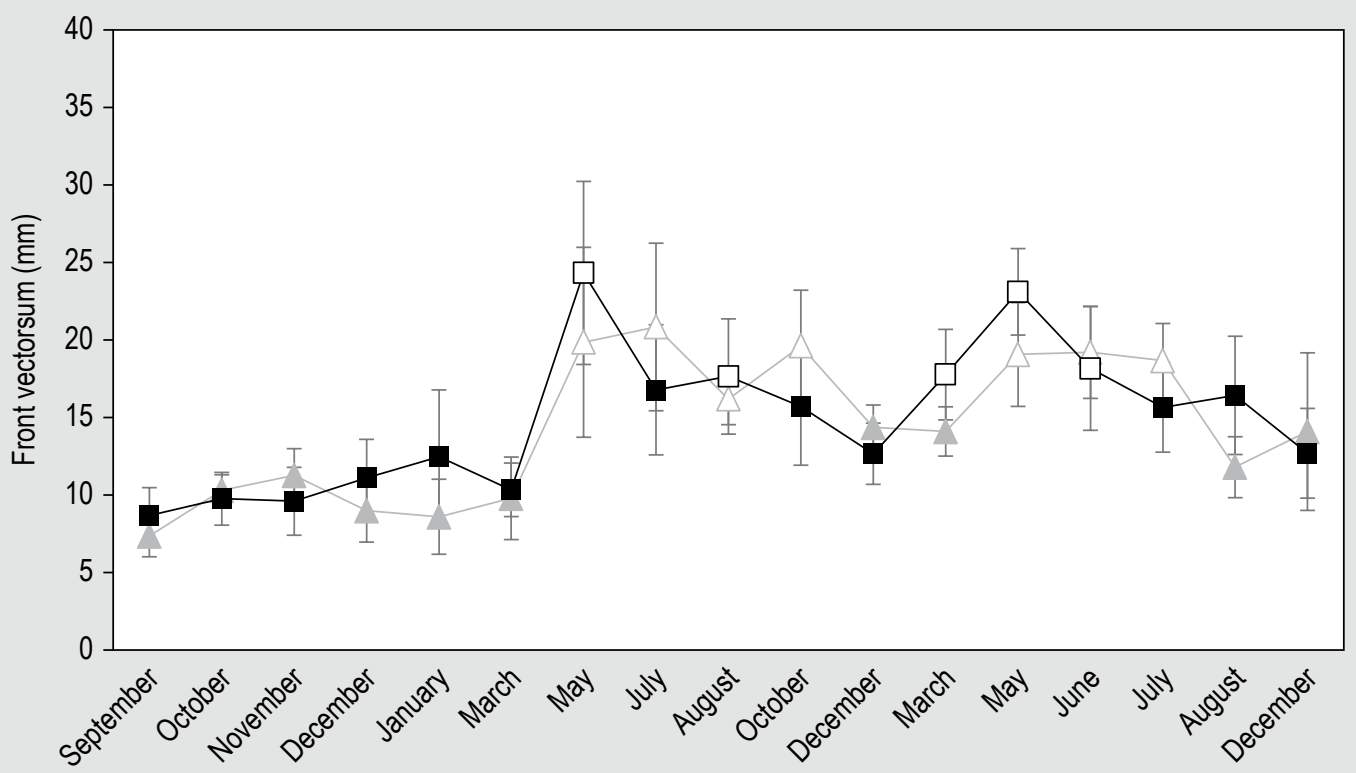

Figure 1. Left-right locomotion asymmetry in front limbs (vector sum (VS) of head sensor) recorded on 17 occasions from age 1 year (start September 2010) to 3 years (end December 2012) in a group of Standardbred trotters kept under controlled conditions. The horses were divided into those that had started in more (M29, triangles) or less (L29, squares) than 29 races by the age of 10 . Values shown are mean \pm standard error. There were no significant differences between the groups. Unfilled icons indicate differences from September 2010 within groups $(P<0.05)$.

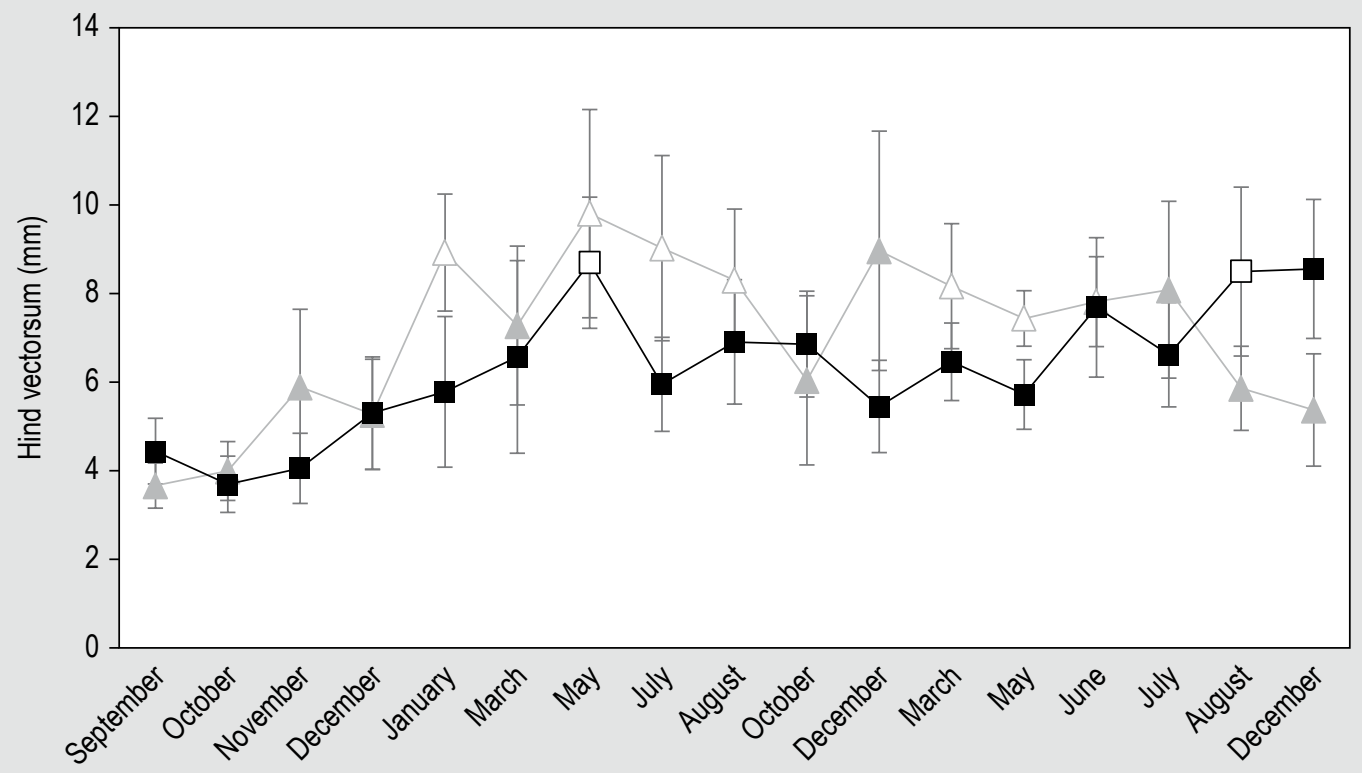

Figure 2. Left-right locomotion asymmetry in hind limbs (vector sum (VS) of pelvic sensor) recorded on 17 occasions from age 1 year (start September 2010) to 3 years (end December 2012) in a group of Standardbred trotters kept under controlled conditions. The horses were divided into those that had started in more (M29, triangles) or less (L29, squares) than 29 races by the age of 10. Values shown are mean \pm standard error. There were no significant differences between the groups. Unfilled icons indicate differences from September 2010 within groups $(P<0.05)$. 
Table 1. Left-right locomotion asymmetry (LSmean \pm standard error, $\mathrm{mm}$ ) in front and hind limbs (vector sum (VS) of head and pelvic sensor, respectively) from July to December at the age of 3 years in a group of Standardbred trotters kept under controlled conditions. ${ }^{1}$

\begin{tabular}{lrrl} 
& \multicolumn{1}{c}{ M29 } & \multicolumn{1}{l}{ L29 } & $P$-value \\
& & & \\
VS front & $15.1 \pm 2.9$ & $15.5 \pm 2.5$ & 0.82 \\
VS hind & $6.6 \pm 1.4$ & $7.8 \pm 1.2$ & 0.50 \\
\hline
\end{tabular}

${ }^{1}$ Horses were divided into those that had started in more or less than 29 races (M29 and L29, respectively), by the age of 10 .

and mean or peak hind and front limb asymmetries (data not shown).

\section{Recordings during trot in hand and in a cart on a track}

The VSs for both front and hind limbs recorded during trot in hand did not differ from VSs recorded on the track $\left(\mathrm{VS}_{\text {front }}\right.$ in hand vs on track, slow and fast: $19 \pm 14,15 \pm 12$ and $12 \pm 8$, respectively, and $\mathrm{VS}_{\text {hind }}$ in hand vs on track slow and fast; $8 \pm 5,7 \pm 4$ and $7 \pm 5$, respectively, $P>0.05)$. On the track, there was also no significant difference $(P>0.05)$ in overall (2.5 years) VS values between the two groups of horses $\left(\mathrm{VS}_{\text {front }} \mathrm{M} 29\right.$ and L29 horses: $14 \pm 10$ and $17 \pm 13$ $\mathrm{mm}$, respectively, $\mathrm{VS}_{\text {hind }} \mathrm{M} 29$ and L29 horses; $8 \pm 5$ and $7 \pm 4 \mathrm{~mm}$, respectively).

\section{Discussion}

The present study reveal that results obtained from regular objective symmetry assessments during trot in hand in young Standardbred horses are not a simple and straightforward predictor of future racing career. Throughout the 2.5 years, there were no overall differences in asymmetry between horses performing more or less races than the median of the cohort born the same year. However, by the end of their 3-year-old seasons, horses that performed more races (M29) had recovered from earlier elevations in hind limb asymmetry while the others (L29) had not. In August as 3-year-olds, these levels were the highest ever recorded. Although symmetry recordings does not say anything about the cause (e.g. pain), we nevertheless suggest that hind limb asymmetry levels around $10 \mathrm{~mm}$

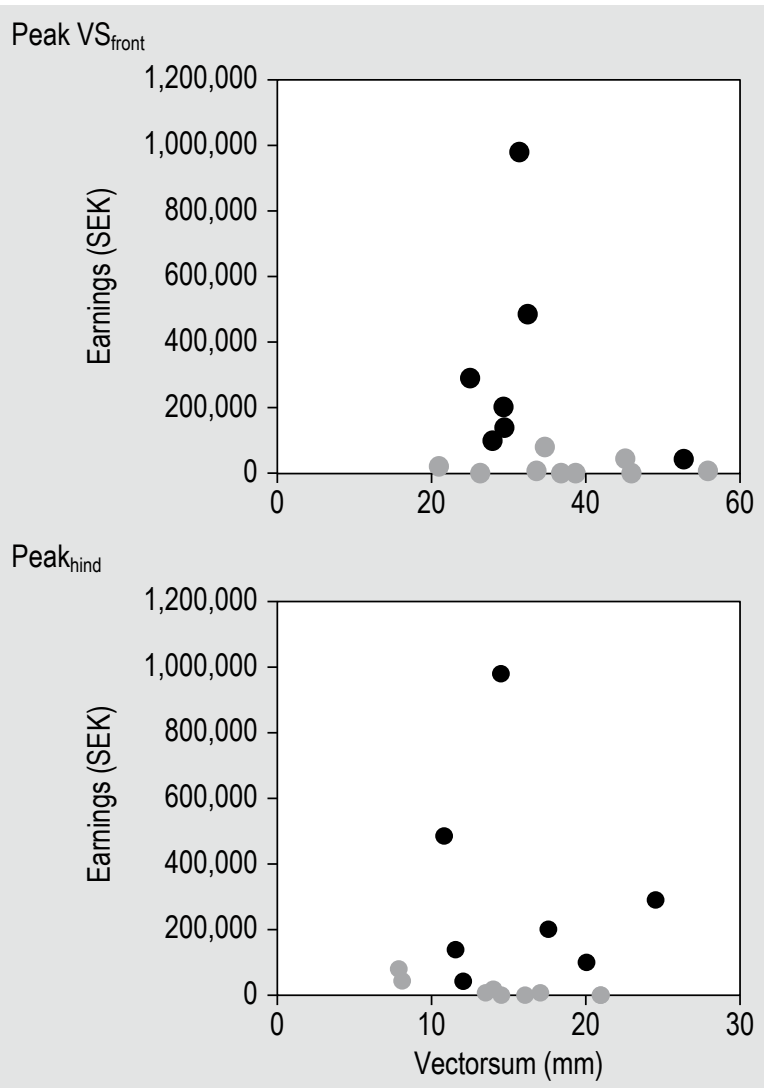

Mean VS front

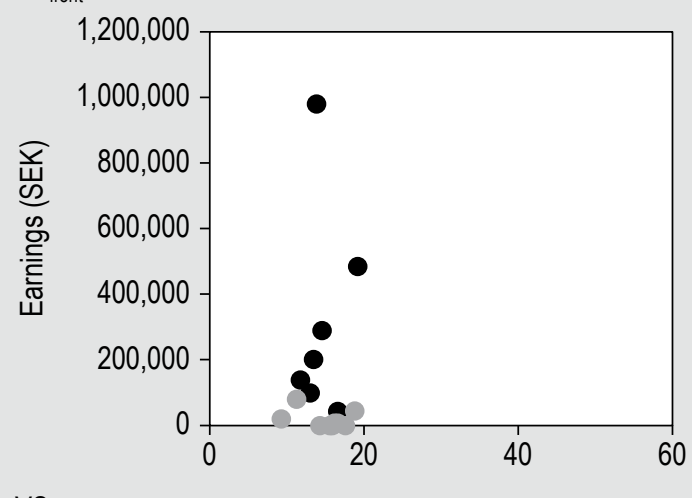

Mean VS $S_{\text {hind }}$

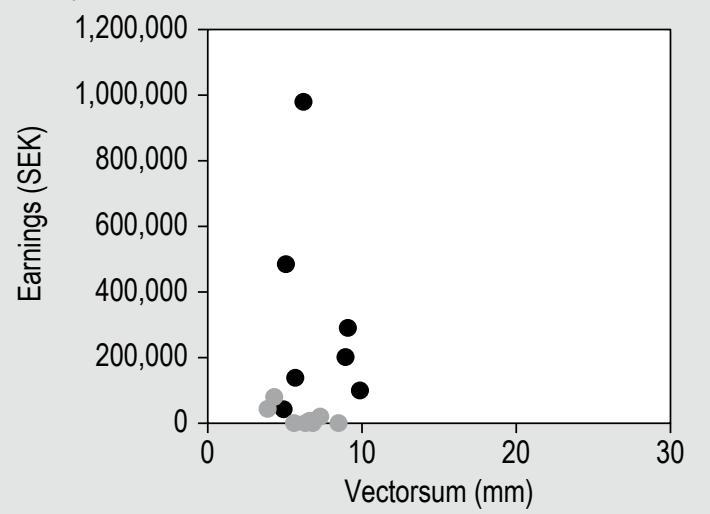

Figure 3. Relationships between individual lifetime earnings (at age 10 years) and individual mean front, mean hind, peak front and peak hind limb asymmetry (vector sum (VS)). Symmetry recordings from 16 Standardbred horses on 17 occasions from age 1 through 3 years of age. Black markers show individuals that raced more times than the median (29 times, M29) of the cohort born the same year. Grey markers show individuals that raced fewer times than the median of the cohort (L29). 
observed during the last five months of the 3-years-season can be a warning of future impaired performance. To our knowledge, this is the first study to link level of asymmetry to a true measure of performance in Standardbred horses.

A possible explanation for the elevated asymmetry observed in L29 horses by the end of the 3-years-season is that by that time the aim is to make the horses competitive in racing, i.e. to increase their maximum speed over race distance. It is logical that it is the hind limb that will react on increased speed since there is a clear connection between increased speed and increased peak load (Crevier Denoix et al., 2014). Increased speed require the horses to perform close to their maximum potential, so those that are not able to adapt to the increased demands and react with increased asymmetry are also likely to be prone to future injuries, and therefore fewer lifetime starts. Occasional observations of asymmetry above $10 \mathrm{~mm}$ seem not to be a concern, however. All horses except two showed hind limb asymmetries greater than $10 \mathrm{~mm}$ (peak ranges: 11-25 $\mathrm{mm}$ ) at least once, but this was not linked at all to lifetime earnings or race record. Another possible explanation for the elevated hind limb asymmetry in L29 horses could be that these horses were subjected to more challenging races. However, race data from the Swedish trotting Association does not support that. In contrast, these horses performed at slower speeds during races, i.e. group L29 were $1.2 \mathrm{~s} /$ $\mathrm{km}$ slower than group M29. It could be speculated that this was due either to (subclinical) lameness or to that L29 horses were less fit or talented. There is, however, no support for these horses being less talented with respect to the genetic background, since the mean pedigree index estimated with the BLUP-method (Best Linear Unbiased Prediction (Swedish Trotting Association, 2021; Ringmark et al., 2015)) did not differ from the mean of M29 horses (data not shown).

There were no indications that front limb asymmetry as a whole was explanatory for the future career. Neither mean nor peak front asymmetry was linked to earnings. However, we have shown previously that the horses which did the qualification race early had less asymmetric front limb locomotion and were less lame in clinical examinations than late qualifiers (Ringmark et al., 2016, 2014). Although this pattern was not significant for the first true race we cannot exclude the possibility that marked front limb asymmetry is associated with low lifetime race participation. In the horses with the absolute poorest lifetime performance (lower than the lower quartile of the cohort, i.e. $<7$ races), the increase in $\mathrm{VS}_{\text {front }}$ in spring as 2-year-olds was double that of the rest of the horses $(33 \pm 16 \mathrm{~mm}$ vs $16 \pm 14 \mathrm{~mm})$ and also three-fold higher than their previous recording (data not shown). Interestingly, this increase in front limb asymmetry co-occurred with a significant increase in workload, since the horses started to do heat training $(1,100-1,600 \mathrm{~m}$ at heart rate $>180$ beats $/ \mathrm{min}$ ) in spring as 2 -year-olds. It can be speculated whether this reaction reflected the ability of the horses to cope with the new increased workload (and perhaps worse coping in horses with $<7$ lifetime races) (Steel et al., 2006) or whether it was due to external factors, such as climate, surface, etc. Mack et al. (2014) have shown that Thoroughbred racehorses may respond with elevated AST (aspartate aminotransferase) levels in response to unaccustomed exercise, indicating increased muscle damage and leakage and maybe some soreness.

There is a known overlap in asymmetry values between horses that are functional and horses that are non-functional and diagnosed with orthopaedic pathology (Hardeman et al., in press; Van Weeren et al., 2017). In this study rather high asymmetry values were seen in some horses and during some periods. This emphasises the importance of professional evaluation of measurements together with other clinical assessment to establish cause of asymmetry. It also implies that it would be an advantage to monitor individual horses over time, using them as their own control, increasing sensitivity and possible early detection of signs of pathology.

Although the conventional lameness examination involves performing trot in hand, one can question its relevance for lameness detection and performance at high-speed trot. To get an indication of the relevance of locomotion asymmetry values in trot in hand to locomotion asymmetry values during driving, we performed additional recordings on a straight track with the horses driven in cart at slow trot and the fastest trot possible for that individual. The results showed that VS for both front and hind limbs recorded in trot in hand did not differ from VS during driving on the track. This is also in accordance with findings by Kallerud et al. (2020). Observations made during trot in hand can therefore be considered useful. The results also showed that there was no significant difference in overall (2.5 years) VS values between horses performing more or less races, which was in agreement with the findings in trot in hand.

Limitations of this study are the small number of horses included and the strong probability that lifetime performance has multiple causes. It was therefore interesting to find indications that changes in locomotion symmetry in young horses in training might be related to future performance. Further studies with a higher frequency of assessment of the horses and with larger groups of horses are therefore warranted. Our results indicated that horses should be monitored, acting as their own controls, and that special attention should be paid when changes in training are introduced. It is probably of higher importance to ensure that the horses can adapt to changes, meaning that a certain amount of asymmetry can be permitted for a period, but then the asymmetry values should approach their normal levels again. To facilitate early detection of health problems, it could also be of value 
to combine measurements of locomotion symmetry with biomarkers indicative of pathological changes and of normal physiological changes, i.e. sound training effects.

\section{Conclusions}

This study showed that regular objective symmetry assessments during trot in hand in young horses do not provide a simple and straight-forward indication of the future racing career. However, we suggest that hind limb asymmetry levels at trot in Standardbred trotters of around $10 \mathrm{~mm}$ (vector sum) by the end of the season as 3-year-olds could be a warning of future impaired performance and measures should be taken to identify the reason.

\section{Acknowledgements}

This work was supported by the Swedish University of Agricultural Sciences, the National Trotting School Wången, Trioplast AB, Dow Chemicals, Swedish Trotting Association, Swedish Horse Racing Totalisator Board and the Swedish Horse Council Foundation. The authors thank all staff and students at Wången and also the reference group of trainers; Stig H Johansson, Thomas Uhrberg and Jan Halberg. The authors also thank the staff at the Swedish Trotting Association for provision of career data, Claudia von Brömmsen for statistical assistance and Mary McAfee for language help.

\section{Conflict of interest}

The authors declare no conflict of interest.

\section{References}

Crevier-Denoix, N., Camus, M., Pourcelot, P., Pauchard, M., Falala, S., Ravary-Plumioen, B., Denoix, J.M., Desquilbet, L. and Chateau, H., 2014. Effect of speed on stride parameters and limb loading: comparison between forelimb and hindlimb at training trot on a firm surface. Equine Veterinary Journal 46: 38. https://doi.org/10.1111/ evj.12267_115

Dyson, P.K., Jackson, B.F., Pfeiffer, D.U. and Price, J.S., 2008. Days lost from training by two- and three-year-old Thoroughbred horses: a survey of seven UK training yards. Equine Veterinary Journal 40: 650-657. https://doi.org/10.2746/042516408X363242

Gaustad, G., Kjærsgaard, P. and Dolvik, N.I., 1996. Lameness in threeyear-old standardbred trotters - influence of parameters determined before three years of age. Journal of Equine Veterinary Science 16: 384-389. https://doi.org/10.1016/S0737-0806(96)80176-6

Hardeman, A.M., Egenvall, A., Serra Bragança, F.M., Koene, M.H.W., Swagemakers, J.H., Roepstorff, L., Van Weeren, P.R. and Byström, A., in press. Movement asymmetries in horses presented for pre purchase or lameness examination. Equine Veterinary Journal. https://doi.org/10.1111/evj.13453
Kallerud, A.S., Fjordbakk, C.T., Hendrickson, E.H.S., Persson-Sjodin, E., Hammarberg, M., Rhodin, M. and Hernlund, E., 2020. Objectively measured movement asymmetry in yearling Standardbred trotters. Equine Veterinary Journal 53: 590-599. https://doi.org/10.1111/ evj.13302

Keegan, K.G., Kramer, J., Yonezawa, Y., Maki, H., Frank Pai, P., Dent, E.V., Kellerman, T.E., Wilson, D.A. and Reed, S.K., 2011. Assessment of repeatability of a wireless, inertial sensor-based lameness evaluation system for horses. American Journal of Veterinary Research 72: 1156-1163. https://doi.org/10.2460/ajvr.72.9.1156

Mack, S.J., Kirkby, K., Malalana, F. and McGowan, C.M., 2014. Elevations in serum muscle enzyme activities in racehorses due to unaccustomed exercise and training. Veterinary Record 174: 145. https://doi.org/10.1136/vr.101669

National Research Council (NRC), 2007. Nutrient requirements of horses ( $6^{\text {th }}$ ed.). National Academy Press, Washington, DC, USA.

Ringmark, S., Jansson, A., Lindholm, A., Hedenström, U. and Roepstorff, L., 2016. A 2.5 year study on health and locomotion symmetry in young Standardbred horses subjected to two levels of high intensity training distance. Veterinary Journal 207: 99-104. https://doi.org/10.1016/j.tvjl.2015.10.052

Ringmark, S., Jansson, A. and Roepstorff, L., 2014. Can locomotion asymmetry in young trotters predict early race performance? Equine Veterinary Journal 46: 47. https://doi.org/10.1111/evj.12267_144

Ringmark, S., Lindholm, A., Hedenström, U., Lindinger, M., Dahlborn, K., Kvart, C. and Jansson, A., 2015. Reduced high intensity training distance had no effect on VLa4 but attenuated heart rate response in 2-3-year-old Standardbred horses. Acta Veterinaria Scandinavica 57: 17. https://doi.org/10.1186/s13028-015-0107-1

Ringmark, S, Revold, T. and Jansson, A., 2017a. Effects of training distance on feed intake, growth, body condition and muscle glycogen content in young Standardbred horses fed a forage-only diet. Animal 11: 1718-1726. https://doi.org/10.1017/S1751731117000593

Ringmark, S., Roepstorff, L., Essén-Gustavsson, B., Revold, T., Lindholm, A., Hedenström, U., Rundgren, M., Ogren, G. and Jansson, A., 2013. Growth, training response and health in Standardbred yearlings fed a forage-only diet. Animal 7: 746-753. https://doi.org/10.1017/S1751731112002261

Ringmark, S., Roepstorff, L., Hedenström, U., Lindholm, A. and Jansson, A., 2017b. Reduced training distance and a forageonly diet did not limit race participation in young Standardbred horses. Comparative Exercise Physiology 13: 265-272. https://doi. org/10.3920/CEP170017

Solé, M., Lindgren, G., Bongcam-Rudloff, E. and Jansson, A., 2020. Benefits and risks of barefoot harness racing in Standardbred trotters. Animal Science Journal 91: e13380. https://doi.org/10.1111/ asj. 13380

Steel, C.M., Hopper, B.J., Richardson, J.L., Alexander, G.R. and Robertson, I.D., 2006. Clinical findings, diagnosis, prevalence and predisposing factors for lameness localised to the middle carpal joint in young Standardbred racehorses. Equine Veterinary Journal 38: 152-157.

Swedish Trotting Association, 2021. Avelsindex. Available at: https:// www.travsport.se/avel-och-uppfodning/ovrigt-om-avel/avelsindex/ 
Van Weeren, P.R., Pfau, T., Rhodin, M., Roepstorff, L., Serra Bragança, F., Weishaupt, M.A., 2017. Do we have to redefine lameness in the era of quantitative gait analysis? Equine Veterinary Journal 49: 567-569. https://doi.org/10.1111/evj.12715
Vigre, H., Chriél, M., Hesselholt, M., Falk-Ronne, J. and Kjar Ersboll, A., 2002. Risk factors for the hazard of lameness in Danish Standardbred trotters. Preventive Veterinary Medicine 56: 105-117. https://doi. org/10.1016/S0167-5877(02)00158-7 